\title{
Hidratación del aluminato tricálcico \\ en presencia de yeso
}

Para el estudio del comportamiento del cemento portland tiene una importancia fundamental el conocimiento de la hidratación del aluminato tricálcico, por ser uno de sus componentes que primero se hidrata y porque se le achacan la mayoría de los defectos que sufre la pasta de cemento; tales son: retracciones, contracciones, fenómenos exotérmicos y sensibilidad a agentes exteriores de tipo químico.

En lo que sigue se estudia por métodos fisicoquímicos la hidratación del aluminato tricálcico junto con el yeso, ya que éste se emplea como regulador del fraguado en los cementos portland.

\section{PARTE EXPERIMENTAL}

El aluminato tricálcico se obtuvo por calefacción a $1.350^{\circ} \mathrm{C}$ de una mezcla estequiométrica de carbonato cálcico y alúmina, moliendo y repitiendo la calefacción hasta ausencia de $\mathrm{C}_{12} \mathrm{~A}_{7}{ }^{-}$y $\mathrm{CaO}$ por difracción de rayos $\mathrm{X}$.

El aluminato tricálcico se molió y pasó por un tamiz de 4.900 mallas, se mezcló con agua $\mathrm{y}$ yeso en las condiciones indicadas en los resultados; la pasta obtenida se situó en el interior de un molde de goma troncocónico y en el interior de la pasta se sumergieron para medir la resistencia eléctrica: dos electrodos laminares de acero inoxidable de forma trapezoidal de $33 \mathrm{~mm}$ de altura, 40 y 45 $\mathrm{mm}$ de bases y separados $13 \mathrm{~mm}$; para medir la temperatura: cinco termopares en serie de cobre-constantán.
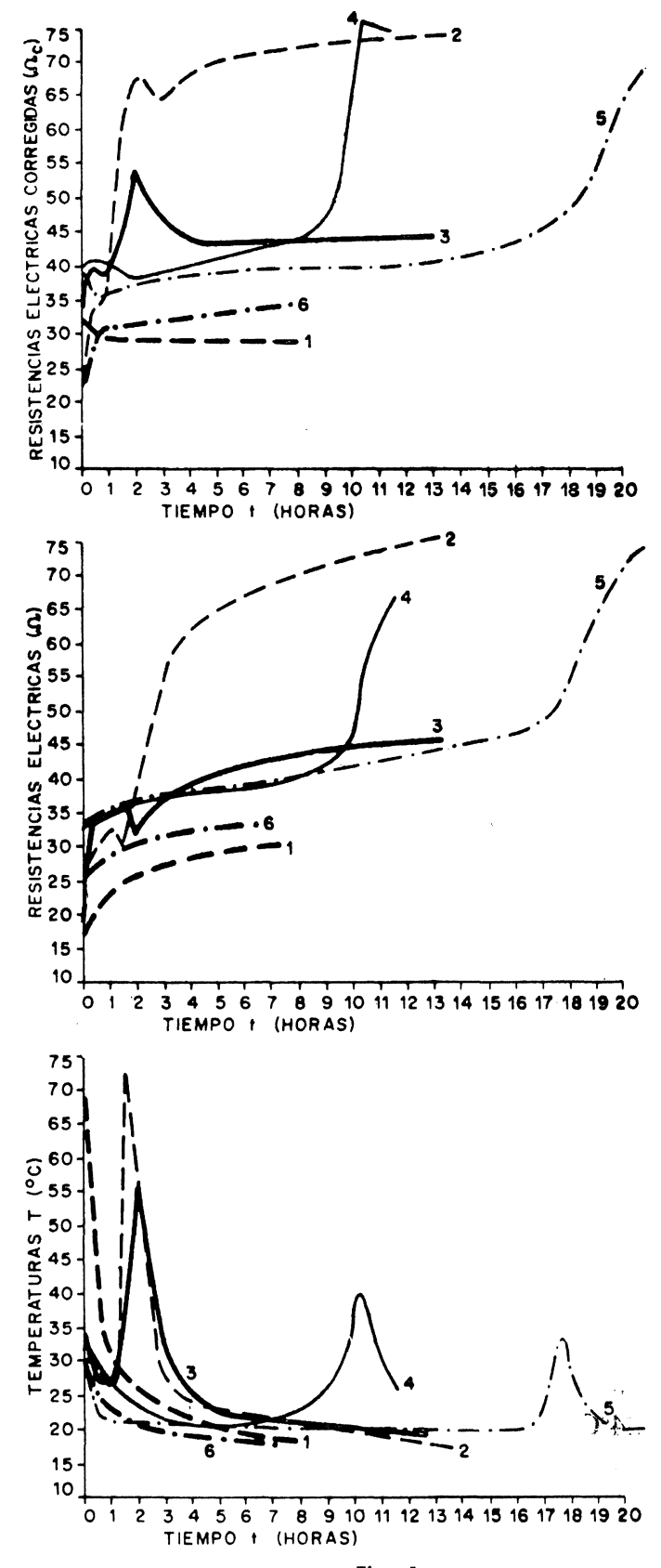

Fig. 1

Se emplea la nomenclatura comúnmente aceptada para la química del cemento: $\mathrm{C}=\mathrm{CaO}, \mathrm{A}=\mathrm{Al}_{2} \mathrm{O}_{3}, \mathrm{~s}=\mathrm{SO}_{3}$ y $\mathrm{H}=\mathrm{H}_{2} \mathrm{O}$. 
Tanto la temperatura como la resistencia eléctrica fueron registradas con un registrador "N-PELEMC" (1).

Después de eliminar la capa superficial de la pasta, se tomó una muestra o más para obtener difractogramas

\section{RESULTADOS}

\section{i) Pasta con $100 \mathrm{~g} \mathrm{C}_{3} \mathrm{~A}+\mathbf{H}$}

La curva que representa la temperatura de la muestra [(fig.1 $)^{-1}$ ] indica que ésta alcanza a los pocos minutos de preparada la pasta temperaturas mayores de $70^{\circ} \mathrm{C}$; a continuación desciende hasta equilibrio con la temperatura ambiente, no experimentando variaciones posteriores.

La curva que representa la resistencia eléctrica de la pasta sube de 17 a 30 ohmios, se estabiliza en este último valor y no varía ulteriormente. La curva corregida indica un descenso en la resistencia que también permanece constante.

El difractograma de la pasta a las 24 horas demuestra que el producto predominante fue aluminato tricálcico hidratado [(fig 2)-a].

El registro gráfico obtenido por espectrografía infrarroja (fig. 3) muestra que en la zona de $3.640 \mathrm{~cm}^{-1}$, correspondiente al grupo $\mathrm{OH}^{-}$, aparece un pico muy intenso, que indica la existencia de hidroxilos en el producto, lo cual nos aclara la probable composición y estructura del $\mathrm{C}_{3} \mathrm{AH}_{6}$ : en él las moléculas de agua no existen como tales, es decir, el aluminato tricálcico hidratado no deberá formularse en forma de óxidos: $3 \mathrm{CaO} \mathrm{Al}_{2} \mathrm{O}_{3} \cdot 6 \mathrm{H}_{2} \mathrm{O}$, como es costumbre en química del cemento, sino en forma de hidróxidos $\mathrm{Ca}_{3}\left[\mathrm{Al}(\mathrm{OH})_{6}\right]_{2}$, formando posiblemente una red mixta. La formulación en forma de mezcla de hidróxidos $\left[2 \mathrm{Al}(\mathrm{OH})_{3} \cdot 3 \mathrm{Ca}(\mathrm{OH})_{2}\right]$ es inadmisible, por cuanto que los difractogramas de rayos $\mathrm{X}$ no revelan la presencia individual de tales hidróxidos.

\section{ii) Pasta con $100 \mathrm{~g} \mathrm{C}_{3} \mathrm{~A}+17 \mathrm{~g} \mathbf{C s H}_{2}+36 \mathrm{~g} \mathrm{H}\left[(\text { fig. } 1)^{-2}\right]$}

La temperatura de la pasta sube a partir de una hora del comienzo de la prueba y alcanza un máximo de unos $70^{\circ} \mathrm{C}$ al cabo de hora y media, descendiendo después hasta equilibrio con la temperatura ambiente.

La resistencia eléctrica sube de 19 a 32 ohmios durante la hora y cuarto desde la preparación de la pasta; el aumento de la temperatura de la pasta provoca un descenso en la resistencia eléctrica aparente de ésta (véase la curva de resistencias corregidas) a un mínimo de 29 ohmios a la hora y media. Alcanza 70 ohmios a las 7 horas y continúa el aumento de la resistencia a razón de 4 ohmios por hora.

\section{iii) Pasta con $100 \mathrm{~g} \mathrm{C}_{3} \mathrm{~A}+17 \mathrm{~g} \mathrm{CsH}_{2}+48 \mathrm{~g} \mathrm{H}\left[(\text { fig. } 1)^{-3}\right]$}

El aumento de agua atenúa los fenómenos del caso anterior: la temperatura inicial de $34^{\circ} \mathrm{C}$ desciende al cabo de 1 hora a $26^{\circ} \mathrm{C}$, sube rápidamente a $57^{\circ} \mathrm{C}$ y se equilibra con la ambiente al cabo de 7 horas.

La resistencia eléctrica aumenta de 25 a 35 ohmios durante hora y media. Coincidiendo con el máximo térmico a las 2 horas, desciende a 31 ohmios y posteriormente aumenta a razón de 1 ohmio cada 3 horas. 


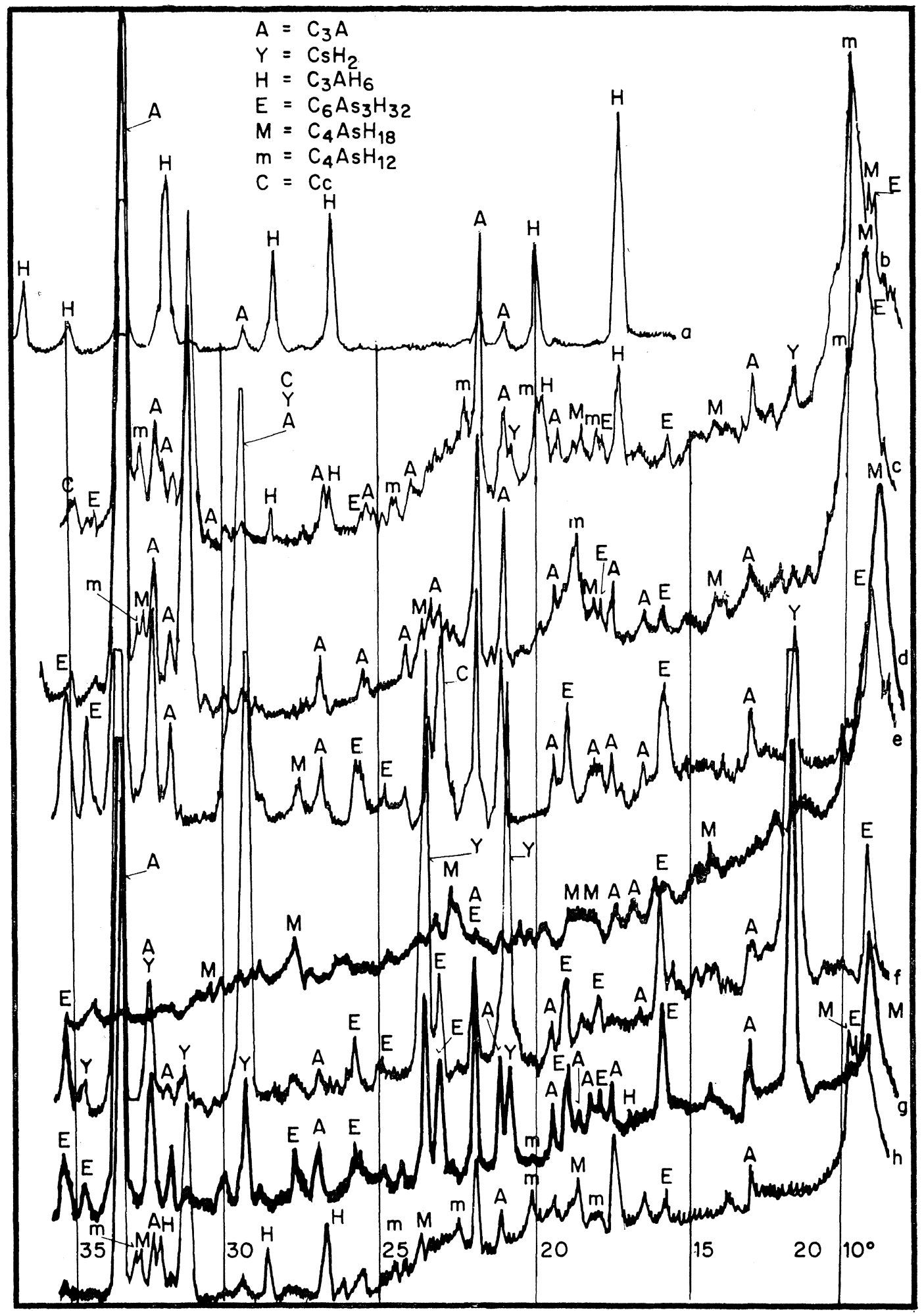

Fig. 2

El difractograma obtenido a las 24 horas [(fig. 2)-b] muestra la casi desaparición del yeso, restos de aluminato tricálcico anhidro, una pequeña cantidad de ettringita y la formación de monosulfoaluminatos con 18, 12 y 7,5 moléculas de agua y de aluminato tricálcico hexahidratado, $\mathrm{Ca}_{3}\left[\mathrm{Al}(\mathrm{OH})_{6}\right]_{2}$. 


\section{iv) Pasta con $100 \mathrm{~g} \mathrm{C}_{3} \mathrm{~A}+25 \mathrm{~g} \mathrm{CsH}_{2}+36 \mathrm{~g} \mathrm{H}\left[(\text { fig.1 })^{-4}\right]$}

La temperatura inicial $\left(30^{\circ} \mathrm{C}\right)$ desciende hasta la ambiente $\left(20^{\circ} \mathrm{C}\right)$ a las 5 horas de comenzada la prueba y sube hasta alcanzar un máximo de $41^{\circ} \mathrm{C}$ a las 10 horas, descendiendo de nuevo hasta equilibrio con la temperatura ambiente.

La resistencia de la pasta sube de 32 a 45 ohmios durante 10 horas. La subida de resistencia se hace más rápida en las 2 horas siguientes, alcanzando un valor de 70 ohmios. El aumento de pendiente coincide con el máximo térmico. Los comienzos de aumento de temperatura y de resistencia eléctrica se dan a las 8 horas. A las 5 horas se da el fin del fraguado según la aguja de Vicat.

El difractograma obtenido a las 24 horas [(fig. 2)-c] muestra: ettringita, aluminato tricálcico anhidro y formación de monosulfoaluminato cálcico con 18 moléculas de agua y ausencia de yeso.

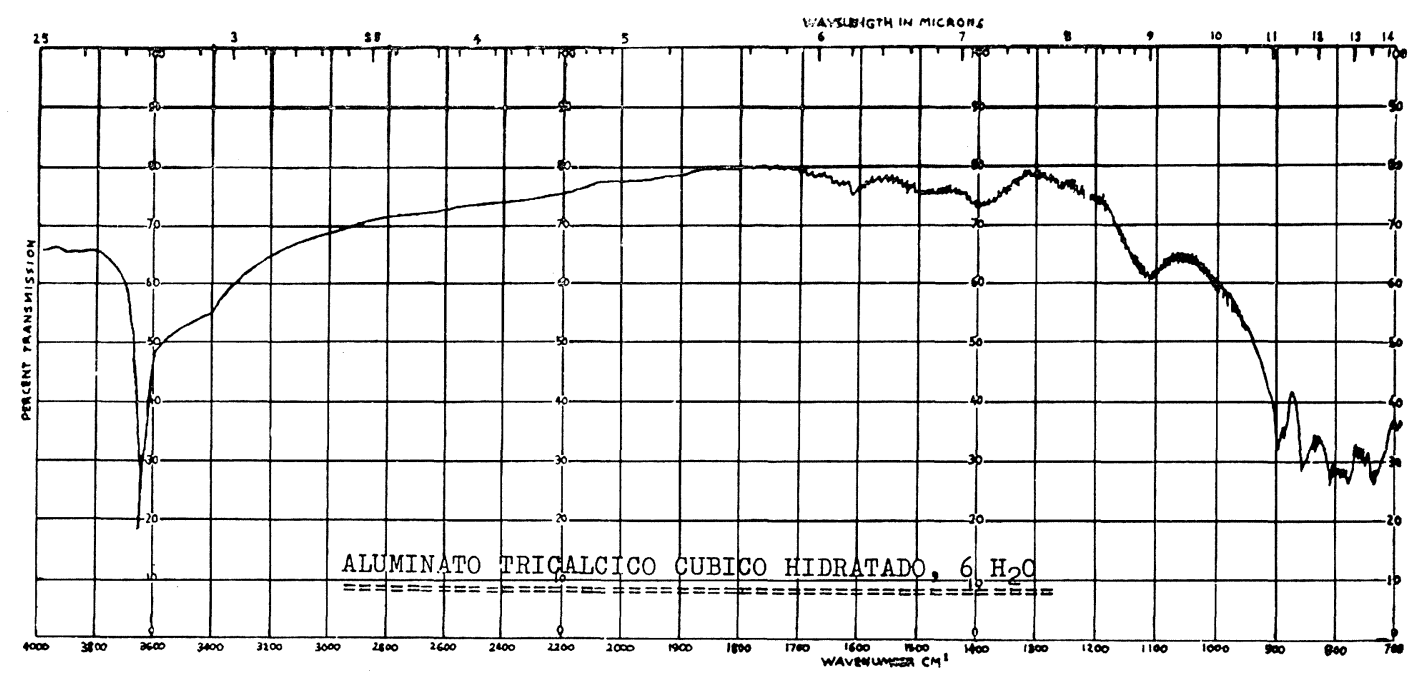

Fig. 3

\section{v) Pasta con 100 g C$_{3} \mathbf{A}+29$ g $\mathbf{C s H}_{2}+36$ g H [(fig. 1) $\left.)^{-5}\right]$}

La temperatura inicial $\left(28^{\circ} \mathrm{C}\right)$ desciende hasta la temperatura ambiente $\left(20^{\circ} \mathrm{C}\right)$ durante las 3 primeras horas; a las 17 horas asciende hasta alcanzar un máximo de $32^{\circ} \mathrm{C}$ a las 18 horas.

La curva que representa la resistencia eléctrica muestra dos tramos: el primero se extiende desde el origen hasta las 15 horas, y el segundo, mas abrupto, desde las 15 horas en adelante, hasta alcanzar 70 ohmios a las 21 horas.

En los difractogramas se observa, en el primero [(fig. 2)-e], la formación de ettringita a la hora y media de preparada la pasta y una fuerte carbonatación, y en el segundo [(figura 2)-d], a las 24 horas de preparada la pasta, se observa la formación de monosulfoaluminatos, principalmente del que tiene 18 moléculas de agua, y desaparece el carbonato cálcico al pulverizar nuevamente la muestra.

\section{vi) Pasta con $100 \mathrm{~g} \mathrm{C}_{3} \mathrm{~A}+63 \mathrm{~g} \mathrm{CsH}_{2}+40 \mathrm{~g} \mathrm{H}\left[(\text { fig. } 1)^{-6}\right]$}

La temperatura inicial de $28^{\circ} \mathrm{C}$ de la pasta desciende, como siempre, hasta equilibrio con la del ambiente, sin variaciones ulteriores en las 24 horas de duración de la prueba. 
La resistencia eléctrica sube desde $20 \mathrm{ohmios}$ al iniciarse la prueba hasta $35 \mathrm{ohmios}$ a las 24 horas de comenzado el registro.

La grafica de difracción [(fig. 2)-f] efectuada a las 16 horas indica que la ettringita formada inicialmente permanece estable.

\section{vii) Compuestos hidratados formados durante la hidratación de pastas que contienen aluminato tricálcico, yeso y agua}

Con una cantidad constante de aluminato tricálcico en cada caso ( $25 \mathrm{~g})$, se prepararon seis muestras, añadiendo: 4,25 g, 5,00 g, 6,25 g, 7,25 g, 9,21 g y 15,75 g de yeso con $12 \mathrm{ml}$ de agua (cuadro I).

Las seis muestras anteriormente descritas se introdujeron en sendos troncos de cono de goma iguales; en contacto con cada muestra se situaron cinco termopares en serie para efectuar simultáneamente el registro térmico en igualdad de condiciones (fig. 4); los máximos térmicos aparecieron a los tiempos indicados en el cuadro I.

El retraso en la aparición del máximo termico en todas las muestras respecto a las estudiadas anteriormente se debe a que en este caso las muestras tenían un volumen menor, lo cual hace que el intercambio térmico muestra-aire se efectúe a mayor velocidad, con lo cual los procesos físico-químicos se retrasan al ser las temperaturas menores.

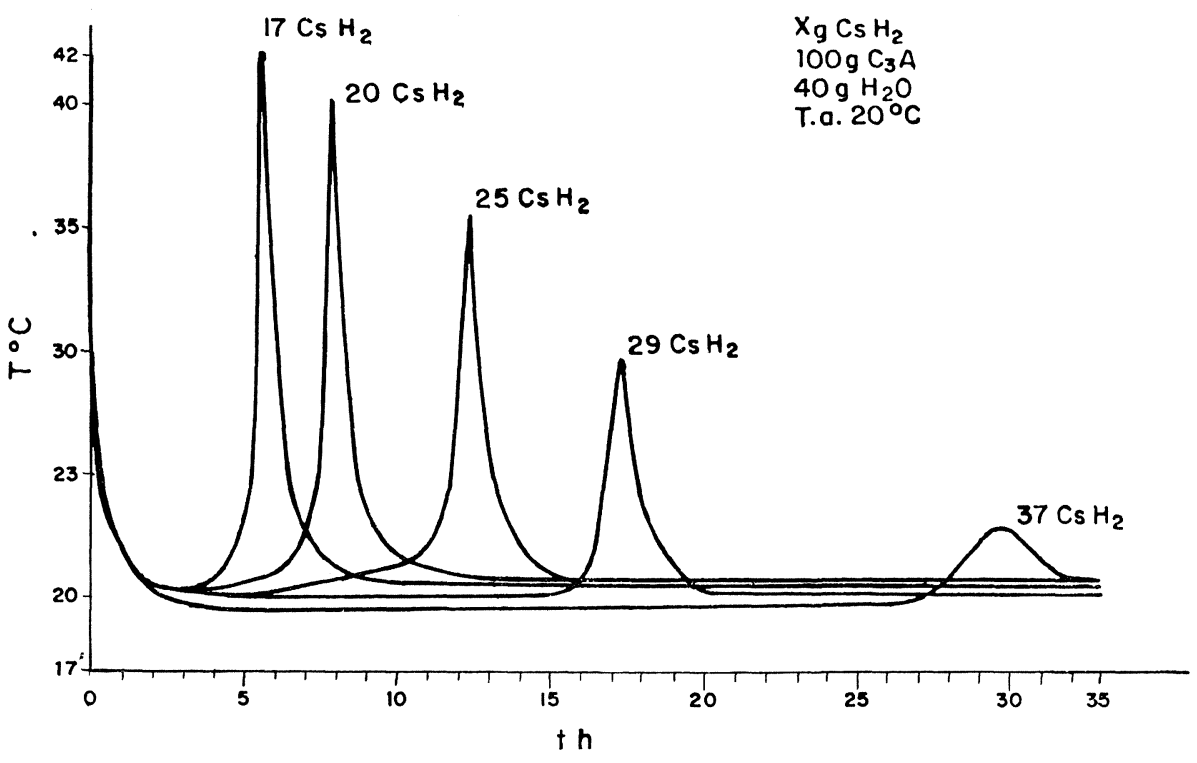

Fig. 4

Los máximos térmicos se produjeron a intervalos de tiempo mayores al aumentar la proporción de yeso en la pasta, y las temperaturas de los máximos fueron disminuyendo al aumentar la proporción de yeso.

La muestra que contenía $5 \mathrm{~g}$ de yeso, como más representativa y por manifestar el máximo térmico relativamente pronto, se registró por difracción de rayos $\mathrm{X}$ durante su hidratación a los tiempos indicados en el cuadro II, en el cual también se indican las intensidades relativas de los picos de varios compuestos; de los difractogramas obtenidos se dan dos [(fig. 2)-g-h $]$. Con los datos del cuadro II se obtuvo la gráfica de la figura 5 , en la cual se aprecia la cinética de la formación y desaparición de los compuestos que intervienen en la hidratación en relación con las variaciones térmicas que experimenta la pasta. 
C U A D R O I

\begin{tabular}{|c|c|c|c|c|c|c|c|}
\hline \multicolumn{2}{|c|}{ Máximo térmico } & \multirow{2}{*}{$-\mathrm{C}_{3} \mathrm{~A}$} & \multirow{2}{*}{$\frac{\mathrm{CsH}_{2}}{\mathrm{~g}}$} & \multirow{2}{*}{$\begin{array}{r}\text { H } \\
\text { g }\end{array}$} & \multirow{2}{*}{$\begin{array}{l}\mathrm{Cs}_{2}-\mathrm{C}_{3} \mathrm{~A} \\
\mathrm{xg}-100 \mathrm{~g}\end{array}$} & \multicolumn{2}{|c|}{$\mathrm{CsH}_{2}-\mathrm{C}_{3} \mathrm{~A}$} \\
\hline h & $\min$ & & & & & $\mathbf{x} \mathbf{M}_{1}$ & $1 \mathrm{M}$ \\
\hline 5 & 30 & 25 & 4,25 & 12 & 17 & 0,27 & \\
\hline 8 & - & 25 & 5,00 & 12 & 20 & 0,31 & \\
\hline 12 & 20 & 25 & 6,25 & 12 & 25 & 0,39 & \\
\hline 17 & 20 & 25 & 7,25 & 12 & 29 & 0,43 & \\
\hline 29 & 40 & 25 & 9,21 & 12 & 37 & 0,50 & \\
\hline 60 & - & 25 & 15,75 & 12 & 63 & 1,00 & \\
\hline
\end{tabular}

C U A D R O II

\begin{tabular}{|c|c|c|c|c|c|c|c|c|c|c|c|}
\hline Compuestos & Fórmula & $2 \theta$ en $\circ$ & $15 \mathrm{~min}$ & $\begin{array}{cc}1 & h \\
30 & \min \end{array}$ & $\begin{aligned} 2 \mathrm{~h} \\
45 \mathrm{~min}\end{aligned}$ & ${ }^{3} 5^{3 ~} \mathrm{~m}$ & $\begin{array}{c}4 \mathrm{~h} \\
30 \mathrm{~min}\end{array}$ & $\begin{array}{cc}5 \mathrm{~h} \\
20 \mathrm{~min}\end{array}$ & $\begin{array}{c}6 \mathrm{~h} \\
20 \mathrm{~min}\end{array}$ & $20^{8 \mathrm{~h}} \mathrm{~min}$ & $24 \mathrm{~h}$ \\
\hline $\begin{array}{l}\text { Yeso } \\
\text { Aluminato tricálcico } \\
\text { Ettringita } \\
\text { Monosulfoaluminato } \\
\text { Monosulfoaluminato } \\
\text { Monosulfoaluminato } \\
\text { Aluminato tricálcico } \\
\text { hidratado } \\
\text { Temperatura }{ }^{\circ} \mathrm{C}\end{array}$ & $\begin{array}{l}\mathrm{Cs}_{\mathrm{H}} \mathrm{H}_{2} \\
\mathrm{C}_{3} \mathrm{~A} \\
\mathrm{C}_{3} \mathrm{~A} \cdot 3 \mathrm{C} \cdot \mathrm{H}_{32} \\
\mathrm{C}_{3} \mathrm{~A} \cdot \mathrm{Cs} \cdot \mathrm{H}_{18} \\
\mathrm{C}_{3} \mathrm{~A} \cdot \mathrm{Cs} \cdot \mathrm{H}_{12} \\
\mathrm{C}_{3} \mathrm{~A} \cdot \mathrm{Cs} \cdot \mathrm{H}_{12-18} \\
\mathrm{C}_{3} \mathrm{~A} \cdot \mathrm{H}_{6}\end{array}$ & $\begin{array}{l}29,0 \\
47,6 \\
15,9 \\
9,2(18,5) \\
9,9(20,0) \\
31,0 \\
17,2\end{array}$ & $\begin{array}{r}85 \\
92 \\
6 \\
- \\
- \\
- \\
- \\
- \\
59\end{array}$ & $\begin{array}{l}64 \\
90 \\
16 \\
- \\
- \\
- \\
- \\
28\end{array}$ & $\begin{array}{l}23 \\
80 \\
20 \\
-(2) \\
- \\
- \\
- \\
28\end{array}$ & $\begin{array}{l}5 \\
72 \\
6 \\
33 \quad(10) \\
- \\
35 \\
- \\
50\end{array}$ & $\begin{array}{l}4 \\
60 \\
4 \\
60 \quad(16) \\
- \\
43 \\
2 \\
100\end{array}$ & $\begin{aligned} & 3 \\
& 40 \\
& 3 \\
& 45(13) \\
& 15(6) \\
& 49 \\
& 10 \\
& 80\end{aligned}$ & $\begin{array}{l}\overline{-} \\
46 \\
\overline{47} \\
28(14) \\
47 \\
\\
19 \\
35\end{array}$ & $\begin{array}{l}\overline{46} \\
\overline{30}(8) \\
20(9) \\
46 \\
20 \\
27\end{array}$ & $\begin{array}{ll}- & \\
50 & \\
- & \\
25 & (5) \\
25 & (9) \\
36 & \\
& \\
20 & \\
22 & \end{array}$ \\
\hline
\end{tabular}




\section{INTERPRETACION}

\section{i) Hidratación del aluminato tricálcico}

Al corregir la resistencia registrada ( $\mathrm{R}$ ohmios) en función de las temperaturas $\mathrm{T}$, teniendo en cuenta que $1^{\circ} \mathrm{C} \simeq 2 \%$ de $\mathrm{R}, \mathrm{y}$ que al aumentar $\mathrm{T}$ disminuye $\mathrm{R}$ (conducción electrolítica-iónica), resulta para la resistencia corregida $R_{c}\left(\Omega_{c}\right)$ [(fig.1 $\left.)^{-1}\right]$ un valor prácticamente constante, lo cual indica dos cosas:

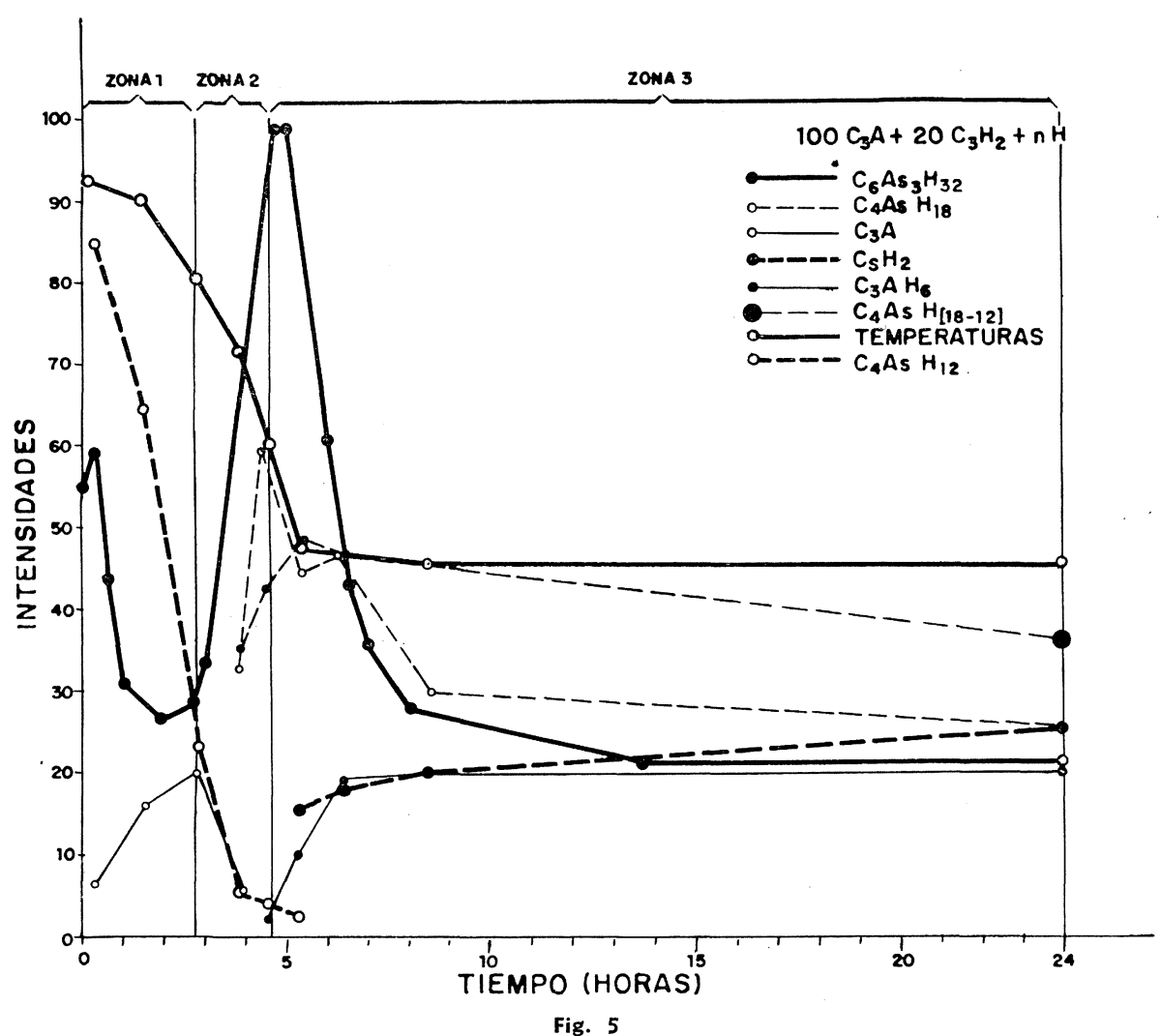

1) $R_{c}$ no varía, por haber terminado el fraguado (fin del fraguado, instantáneo).

2) La variación de $R$ en la figura es sólo consecuencia de la variación de $T$.

Al disminuir $T$ disminuye $R_{c}\left(\Omega_{c}\right)$ en la curva corregida y sube $R(\Omega)$ en la curva sin corregir, ya que aparecen iones móviles, $\mathrm{OH}^{-}$, en la fase líquida, según lo confirma la gráfica de infrarrojos de la figura 3 , la cual demuestra la existencia de $\mathrm{OH}^{-}$como tales en la fase sólida que, de acuerdo con Volant (2), tiene la fórmula $\mathrm{Ca}_{3}\left[\mathrm{Al}(\mathrm{OH})_{6}\right]_{2}$.

\section{ii) Hidratación de $100 \mathrm{~g} \mathrm{C}_{3} \mathrm{~A}+17 \mathrm{~g} \mathrm{CsH}_{2}+\mathrm{H}$}

En la [(fig. 1)-2], al aumentar $\mathrm{T}$ aumentan las resistencia $\mathrm{R}(\Omega)$ y $\mathrm{R}_{\mathrm{c}}\left(\Omega_{\mathrm{c}}\right)$, de acuerdo con los resultados de la figura 5 , en la cual la curva T-t presenta un efecto exotérmico muy al principio, seguido de un enfriamiento espontáneo y de una nueva subida de $\mathrm{T}$ debida a la formación de monosulfoaluminatos y a la hidratación directa del $\mathrm{C}_{3} \mathrm{~A}$. Es posible así observar tres fases, según el siguiente esquema:

Primera fase:

$$
\mathrm{C}_{3} \mathrm{~A}+3 \mathrm{CsH}_{2}+26 \mathrm{H} \rightarrow \mathrm{C}_{6} \mathrm{As}_{3} \mathrm{H}_{32}+\Delta
$$


Segunda fase:

$$
\mathrm{C}_{6} \mathrm{As}_{3} \mathrm{H}_{32}+2 \mathrm{C}_{3} \mathrm{~A}+22 \mathrm{H} \rightarrow 3 \mathrm{C}_{4} \mathrm{AsH}_{18}+2 \Delta
$$

Tercera fase:

$$
\mathrm{C}_{4} \mathrm{AsH}_{18}+\mathrm{C}_{3} \mathrm{~A} \rightarrow \mathrm{C}_{4} \mathrm{AsH}_{(12-7)}+\mathrm{C}_{3} \mathrm{AH}_{6}+\Delta
$$

Esto prueba que hay que interpretar la hidratación del aluminato tricálcico en presencia de yeso y en ausencia de hidróxido cálcico, en el sentido de que la fase final estable sulfatada es el monosulfoaluminato y la fase final estable alumínica es el aluminato tricálcico hexahidratado cúbico.

\section{iii) Hidratación de $100 \mathrm{~g} \mathrm{C}_{3} \mathrm{~A}+17 \mathrm{~g} \mathrm{CsH}+48 \mathrm{~g} \mathrm{H}$ [(fig. 1) $\left.)^{-3}\right]$}

Las diferencias de estas figuras con la $1^{-2}$ son: un máximo de T más bajo y que la segunda rama de $R$-t $(\Omega)$ es menos abrupta y con otra convexidad.

En consecuencia, la curva $R_{c}$-t $\left(\Omega_{c}\right)$ presenta un máximo que coincide con el mínimo de la curva R-t $(\Omega)$ (no corregida) y con el máximo de la curva T-t.

La primera rama de $R_{c}-t\left(\Omega_{c}\right)$ da mayores valores de resistencias correspondientes a una mayor cantidad de agua en la pasta. Con relación a la [(fig. 1)-2], se retrasa la hidratación. Si hay transformación de ettringita en monosulfoaluminatos y éstos se degradan perdiendo agua, ésta, al quedar libre, puede hacer más conductora la pasta y crecer menos rápidamente $R_{\mathrm{c}}\left(\Omega_{\mathrm{c}}\right)$. Unido a lo anterior (mayor cantidad de agua de amasado) y al curso similar de $\mathrm{T}$ en cuanto a la forma, se puede explicar el máximo de $R_{c}(\Omega)$. Sobre todo si el agua liberada se emplea en formar otros aluminatos hidratados [(fig. 2)-b] que movilizan $\mathrm{OH}^{-}$(fig. 3), como así sucede.

Hasta las 2 horas de hidratación, transcurren las curvas como en la [(fig.1)-2]; la diferencia está después de las 2 primeras horas de hidratación, ya que la $R_{c}$ baja continúa notablemente (en vez de estabilizarse o subir), y la $R(\Omega$ ), en vez de subir abruptamente a partir del mínimo, lo hace de una forma más suave. Al no ser esto explicable por solo efecto de T, hay que buscar la razón en las diferencias de las condiciones experimentales correspondientes a las figuras $1^{-2}$ y $1^{-3}$ : el agua de amasado es un $33 \%$ mayor en el caso de la figura $1^{-3}$.

Después de las 2 primeras horas de hidratación, se produce un proceso (distinto en ambas figuras y mucho más intenso en la $1^{-3}$ que en la $1^{-2}$ ) que provoca un aumento de conductividad.

Este proceso se interpreta como la transformación, a partir del máximo de T, del monosulfoaluminato con 18 moléculas de agua en el monosulfoaluminato con 12 moléculas de agua y aún menos (1,5 moléculas), con cesión de $6 \mathrm{H}_{2} \mathrm{O}$ por mol de monosulfoaluminato por lo menos; agua que se emplea en la formación de $\mathrm{C}_{3} \mathrm{AH}_{6}$ en la tercera fase, como indica la figura 5.

$\mathrm{El} \mathrm{C}_{3} \mathrm{AH}_{6}$ formado moviliza o libera iones $\mathrm{OH}^{-}$que provocan un aumento de conductividad, con caída de la resistencia corregida $\left(\Omega_{\mathrm{c}}\right)$ en forma similar y hasta valores análogos a los de la figura $1^{-1}\left(\Omega_{\mathrm{c}}\right.$ constante y más bajo).

\section{iv) Hidratación de $100 \mathrm{~g} \mathrm{C}_{3} \mathrm{~A}+25 \mathrm{~g} \mathrm{CsH}_{2}+36 \mathrm{~g} \mathrm{H}\left[(\text { fig. } 1)^{-4}\right]$}

Los máximos de la resistencia eléctrica corregida $\left(\Omega_{\mathrm{c}}\right)$ y de la temperatura $(\mathrm{T})$ aparecen después de 10 horas, indicando un retraso notable en la hidratación (fase segunda), debido a la mayor cantidad de yeso y a la menor cantidad de agua (mayor concentración de $\mathrm{SO}_{4}=$ en la fase líquida). 
Esto, unido a la ausencia de $\mathrm{C}_{3} \mathrm{AH}_{6}$ detectable por rayos $\mathrm{X}$ incluso a las 24 horas, tal como ponen de relieve las figuras $\left(2^{-c}\right.$ y 5$)$, parece indicar que el yeso es suficiente para formar sólo trisulfoaluminato hexacálcico, protector y retardador del fraguado, por un mecanismo análogo al del esquema del punto ii) de la interpretación en su fase primera. A partir de los máximos de la resistencia corregida $\left(R_{c}\right)$ y de $T$, tiene lugar la fase segunda, según dicho esquema, sin que en este caso se dé la fase tercera, por lo menos hasta mucho después de las 24 horas.

Parece ser que si se forma $\mathrm{C}_{3} \mathrm{AH}_{6}$, éste es submicrocristalino y no se detecta por rayos X. Dado que el efecto térmico consiguiente al desdoblamiento de la ettringita en los monosulfoaluminatos suele ir acompañado del efecto térmico de la formación tardía de $\mathrm{C}_{3} \mathrm{AH}_{6}$, es posibla que a partir de las 10-11 horas se forme una ligera cantidad de esta fase, que será la responsable de la disminución de la resistencia corregida $\left(\Omega_{c}\right)$ después del máximo térmico.

Por otra parte, complementa esta interpretación el hecho de que los monosulfoaluminatos son más estables que la ettringita cuando la temperatura se eleva, condición en la que también se forma y es estable el $\mathrm{C}_{3} \mathrm{AH}_{6}$ cúbico (frente al $\mathrm{C}_{4} \mathrm{AH}_{13}$ hexagonal, estable a más baja temperatura). Todo esto encaja perfectamente con la forma de la curva T-t y el valor del máximo de la misma correspondiente a las 10 horas, en la figura $1^{-4}$.

\section{v) Hidratación de $100 \mathrm{~g} \mathrm{C} \mathbf{C}_{3} \mathbf{A} 29 \mathbf{g ~ C s H}_{2}+36 \mathrm{~g} \mathbf{H}\left[(\text { fig. 1) })^{-5}\right]$}

Los máximos de $R_{c}$ y $T$ aparecen después de las 18 horas, aumentando aún más el retraso en la hidratación (fase segunda) por el aumento de la cantidad de yeso y la consiguiente disminución de agua.

La fase tercera se retrasará aún más en esta pasta, pues las temperaturas del segundo máximo son menores, lo cual indica menor formación de monosulfoaluminatos y, por consiguiente, también de $\mathrm{C}_{3} \mathrm{AH}_{6}$, no detectable por difracción de rayos $\mathrm{X}$ a las 24 horas.

Ello se debe a una menor velocidad de descomposición de la ettringita, como consecuencia de una menor elevación de la temperatura en el segundo máximo. Por lo demás, la interpretación es análoga a la del caso precedente.

\section{vi) Hidratación de $100 \mathrm{~g} \mathrm{C}_{3} \mathrm{~A}(1 \mathrm{~mol})+63 \mathrm{~g} \mathrm{CsH}_{2}(1 \mathrm{~mol})+40 \mathrm{~g} \mathrm{H}$}

En este caso la formación de ettringita es lentísima, sin que se desdoble en monosulfoaluminatos ni aparezca, por tanto, el máximo de $\mathrm{T}$ antes de las 24 horas.

Esta corresponde en la figura 5 a la curva de $\mathrm{C}_{6} \mathrm{As}_{3} \mathrm{H}_{32}$, aunque allí la proporción de yeso era mucho menor $(20 \mathrm{~g})$ y aparecía el máximo de monosulfoaluminato a poco menos de 5 horas (después de haber comenzado a disminuir la ettringita). Como en el caso presente no baja la ettringita, tampoco sube el monosulfoaluminato ni se da el correspondiente efecto térmico con un máximo de $\mathrm{T}$, ya que existe un exceso de yeso libre en la pasta, el cual estabiliza la ettringita.

Los bajos valores de resistencia eléctrica obtenidos en esta muestra son causados por no haber terminado aún el proceso de formación de ettringita y por lo tanto, la fase líquida aún tiene $\mathrm{SO}_{4}=$ conductores. 


\section{vii) Compuestos hidratados formados durante la hidratación de pastas que contienen $\mathrm{C}_{3} \mathrm{~A}, \mathrm{CsH}_{2}$ y $\mathrm{H}$}

Desde el comienzo de la hidratación hasta las 3 horas, se observa en la zona 1 de la figura 5 un primer ciclo térmico en correspondencia con la formación de ettringita y según la primera fase del punto ii) de la interpretación.

En la zona segunda de la gráfica comienza un segundo ciclo térmico con valores más elevados de temperatura, unido a una disminución en las intensidades de los picos de difracción de rayos $\mathrm{X}$ correspondientes: a la ettringita, al aluminato tricálcico (que desaparece aún más rápidamente que en la zona primera) y al yeso, que se elimina en su totalidad, mientras se forma monosulfoaluminato cálcico con 18 moléculas de agua y conforme a la segunda fase del punto ii).

En la tercera zona continúa la hidratación del aluminato tricálcico aún más acelerada; se transforma el monosulfoaluminato hidratado con 18 moléculas de agua en otro con 12 moléculas, y se forma aluminato tricálcico hidratado cúbico, posiblemente a partir de la descomposición del monosulfoaluminato cálcico menos hidratado, ya que en algunas ocasiones (3) se ha comprobado la aparición simultánea de pequeñas cantidades de yeso.

La formación de monosulfoaluminatos se hace a expensas de la ettringita y de doble cantidad de aluminato tricálcico, que la correspondiente a la propia ettringita, junto con la hidratación del aluminato tricálcico, explican la fuerte reacción exotérmica, que a su vez contribuye a la formación del aluminato tricálcico hidratado.

Las dos reacciones exotérmicas provocadas por la combinación del aluminato tricálcico, para formar ettringita primero y monosulfoaluminato después, dan origen a dos de los ciclos térmicos que se observan en los cementos portland, aunque con las siguientes salvedades:

El primer ciclo térmico corresponde a la formación de ettringita, mientras que el segundo puede corresponder sólo a la formación de monosulfoaluminatos, si por haber poco yeso anteriormente se combinó la totalidad del yeso soluble. En este caso el segundo ciclo puede ser también ocasionado por la hidratación conjunta o simultánea de la alita.

Si hay suficiente yeso en disolución, puede retrasarse el segundo ciclo de hidratación de los sulfoaluminatos hasta la desaparición de dicho yeso. En este otro caso, el segundo ciclo, que otras veces era ocasionado por la hidratación conjunta de los sulfoaluminatos y de la alita, se desdobla en dos, quedando como segundo el de la alita y pasando el de los monosulfoaluminatos a ser un tercer ciclo, tal como describe Lerch (4) en algunos casos de cementos estudiados por él.

La velocidad de reacción es aumentada por temperaturas crecientes; de esta forma, los ciclos térmicos causados por el aluminato tricálcico influyen sobre las velocidades de reacción e hidratación de la alita y del silicato bicálcico, aunque en menor proporción sobre este último, ya que su pequeño contenido en el clínker y su baja reactividad hacen sentir menos la influencia térmica en relación a otras fases del clínker.

\section{O N C L U S I O N E S}

1) Al aumentar la concentración en yeso disminuye la intensidad de los dos máximos térmicos y se retrasa la aparición del segundo máximo.

2) El calor desprendido durante el primer máximo térmico se puede atribuir a la formación de la ettringita. El calor producido durante el segundo máximo es debido a la formación de monosulfoaluminato tetracálcico hidratado y a la hidratación simultánea del aluminato tricálcico. 
3) La presencia de yeso inhibe la formación brusca de monosulfoaluminato y la hidratación del aluminato tricálcico; por tanto, estabiliza a la ettringita.

4) El descenso de resistencia eléctrica es producido por la aparición de iones $\mathrm{OH}$, procedentes del aluminato tricálcico hidratado que se forma.

5) El aumento de la resistencia eléctrica se debe a la formación de monosulfoaluminatos, que eliminan el agua de la pasta.

6) La formación de sulfoaluminatos transcurre de la siguiente manera:

El sulfato cálcico disuelto reacciona con la disolución de aluminatos, que rodea a las partículas de aluminato tricálcico, produsiendo una capa de ettringita que las recubre. La ettringita reacciona con el aluminato, el que recubre dando monosulfoaluminato tetracálcico expansivo, que rompe la cubierta ettringítica. Si hay yeso presente en disolución, se forma más ettringita por reacción con el aluminato tricálcico accesible y con los monosulfoaluminatos. El proceso continúa hasta que se consume el yeso o el aluminato tricálcico; si es el primero el que se agota, se forman muy rápidamente monosulfoaluminatos, dando origen al segundo ciclo térmico; si es el segundo el que se consume, la ettringita formada permanece estable.

En los cementos portland, la presencia de hidróxido cálcico provoca la formación de aluminato tetracálcico hidratado en vez de aluminato tricálcico hidratado; en los cementos puzolánicos puede, en cambio, darse un proceso similar al indicado en el anterior, y, de hecho, así lo hemos encontrado.

\section{B I B L I O G R A F I A}

(1) F. TRIviño.: ION XXXI, 231-235 (1971).

(2) J. Volant.: “Revue des Matériaux de Construction”, número 632, pág. 194 (1968).

(3) F. TRIviño.: Tesis Doctoral (1970).

(4) W. LeRch.: P. C. A. Res. Depto. Bull. (1942). 